Running Head: ECHO-CHAMBERS AND ONLINE MEDIA

Echo-chambers in online news consumption: Evidence from survey and navigation data

\author{
in Spain \\ Ana S Cardenal \\ Universitat Oberta de Catalunya, Spain \\ Carlos Aguilar-Paredes and Camilo Cristancho \\ Universitat de Barcelona, Spain \\ Sílvia Majó-Vázquez \\ University of Oxford, UK
}

\begin{abstract}
Whether people live in echo-chambers when they consume political information online has been the subject of much academic and public debate. This paper contributes to this debate combining survey and web-tracking online data from Spain, a country known for its high political parallelism. We find that users spend more time in outlets of their political leanings but, generally, they engage in considerable cross-partisan media exposure, especially those in the left. Additionally, we use a quasi experiment to test how major news events affect regular patterns of news consumption, and particularly, selective exposure. We find that the nature of news explains changes in users' overall consumption behaviour, but this has less to do with the type of event than with the interest it arises. More importantly, we find that users become more polarized along party lines as the level of news consumption and interest for news increases.
\end{abstract}

Keywords: echo-chambers, news consumption, online media, selective exposure, fragmentation. 


\section{Echo-Chambers in Online News Consumption: Evidence from Survey and Navigation Data in Spain}

The question of whether people live in echo-chambers when they consume political information online has been the subject of much academic and public debate. Echo-chambers have attracted so much attention because they are seen to limit the diversity of opinions people are exposed to, and thus to have adverse effects on the democratic debate, open-mindedness and a healthy public sphere (Pariser, 2011; Sunstein, 2009; Zuiderveen Borgesius, F., Trilling, D., Moeller, J., Bodó, B., de Vreese, C. H., \& Helberger, N., 2016). Observers attribute to echo-chambers a wide range of phenomena that today threaten a healthy democratic public life, such as increasing group polarization, audience fragmentation, and more recently, the circulation of fakenews and misinformation (Allcott \& Gentzkow, 2017; Bennett \& Iyengar, 2008; Chaffee \& Metzger, 2001; Del Vicario et al., 2016).

Digital technologies have been blamed for the emergence of echo-chambers because they have increased the amount of choice in news outlets and facilitated the personalization and filtering of content. The availability of news outlets of a wide range of ideological views together with web-affordances to personalize contents have led many to expect that people will consume only like-minded political news, leading them to inhabit partisan echo-chambers (e.g., Bennett \& Iyengar, 2008; Stroud, 2011). According to some proponents of this view, with the expansion of choice and the increasing possibility of filtering and personalizing online contents, consumers will be motivated to "wall themselves off from topics and opinions that they would prefer to avoid" (Sunstein, 2001, as cited in Bennet and Iyengar, 2008, pp. 201-202). Scholars have also warned about the risks of algorithm curation for involuntary exposure to likeminded information (Pariser, 2011). 
Despite the influence of the partisan echo-chamber hypothesis, the evidence supporting it is scarce. Existing evidence on the role of algorithm curation in crossideological exposure shows that its effect on diversity reduction is small (e.g., Bakshy, Messing, \& Adamic, 2015). Also, evidence of partisan selective exposure to news is at best mixed (Guess, Nyhan, Lyons \& Reifler, 2018).

One reason the echo-chamber hypothesis has gained so much influence in the literature is that it provides a straightforward explanation for phenomena we find hard to explain, such as Brexit or the Trump election (Nelson \& Webster, 2017). Another reason lays in data limitations. Most studies finding support for selective exposure rely on survey data or experimental designs, which suffer from internal and external validity problems that hinder our ability to learn about people's news consumption behaviour in real life situations (Wojcieszak, Bimber, Feldman, \& Stroud, 2016). Although recent research overcomes some of these problems by relying on direct measures of exposure and more comprehensive research designs, most of these studies are based on the US context, which has quite an exceptional media and political system (Valeriani \& Vaccari, 2015).

In this paper, following the work of other pioneering scholars (e.g., DvirGvirsman et al., 2016; Flaxman et al., 2016; Gentzkow \& Shapiro, 2011) we rely on a comprehensive data strategy that combines survey and web-tracking data to test the partisan echo-chambers hypothesis in a non-US context. We use a two-wave panel survey to study the level of ideological segregation in the online news domain in Spain and combine this information with browsing data from the same panel. Focusing on Spain not only adds a comparative dimension to a debate that has been for the most part US-based, but also provides a unique opportunity to submit the partisan selective 
exposure to a tougher test given the polarized nature of the Spanish media system (Hallin \& Mancini, 2004).

Another contribution of this paper is studying selective exposure in situations where news consumption intensifies. We use a quasi experiment to explore how regular patterns of news consumption are altered under external shocks, i.e., when major news events take place. We find that under normal conditions people tend to engage in extensive cross-partisan media consumption, although they still spend more time in congenial partisan media. However, as news consumption increases users tend to become more polarized.

\section{How prevalent are Partisan Echo-Chambers?}

The partisan echo-chamber hypothesis predicts that in a high-choice media environment with many opportunities to personalize contents people will only consume information consistent with their pre-existing political views. This hypothesis has been based mainly on two tenants. The first is structural and related to the personalization of information that results from different curation processes (Thorson \& Wells, 2015), and particularly, algorithmic curation online (Pariser, 2011). The second is based on selective exposure, a foundational concept in studies of communication, which refers to an individual motivation to select like-minded information in order to reduce cognitive dissonance (Festinger, 1957) or defend one's original beliefs (Taber \& Lodge, 2006). This mechanism differs from the previous one because exposure to politically congenial information results from personal choice and is thus behavioural. Others have referred to these two mechanisms in different ways. For example, Zuiderveen Borgesius, F. et al. (2016) refer to selective exposure as "self-selected personalization" and to algorithm 
curation as "pre-selected personalization", and Thurman \& Schifferes (2012), as explicit and implicit personalization.

Algorithms may lock people in information cocoons or echo-chambers without them knowing because they select information based on their past choices and tastes. This, in turn, may create "a unique universe of information for each of us", what has been coined "filter bubbles" (e.g. Pariser, 2011). Even though the filter bubble argument has been quite popular, the evidence supporting it is scant. For example, with regard to the personalization that may result from using search engines, some studies have estimated by $11 \%$ the Google searches that differ due to personalization (Hannak et al., 2013). In an important study examining the effect of News Feed algorithm in exposure to ideologically diverse news articles in Facebook, Bakshy et al., (2015) found that personal choice was more important in reducing cross-cutting exposure than news feed algorithm, and that overall these effects were small.

Partisan echo-chambers however may also result from people's decision to consume only congenial information. According to mainstream accounts of selective exposure, people prefer to consume like-minded political information to avoid cognitive dissonance - i.e., the mental discomfort resulting from being exposed to points of views that contradict their own (Festinger, 1957). Other accounts explain selective exposure according to the theory of motivated reasoning (e.g., Nelson \& Webster, 2017), which assumes that people are motivated to defend their own political views (Taber \& Lodge, 2006). Although there is proof of people's proclivity to choose sources and information that conform to their pre-existing political worldviews, most evidence of selective exposure in online news consumption comes from studies using survey and experimental data (Hollander, 2008; Iyengar \& Hahn, 2009; Knobloch-Westerwick \& Meng, 2009; Kobayashi \& Ikeda, 2009; Stroud, 2011). In contrast, research using 
digital trace data to study peoples' online media diets reveals no evidence of partisan echo-chambers (Dvir-Gvirsman, Tsfati, \& Menchen-Trevino, 2016; Flaxman, Goel, \& Rao, 2016; Gentzkow \& Shapiro, 2011; Guess, 2016; Nelson \& Webster, 2017; Weeks, Ksiazek, \& Holbert, 2016). These mixed results suggest that selective exposure might work in more complex and subtle ways (Holbert, Hmielowski, \& Weeks, 2012; Metzger, Hartsell, \& Flanagin, 2015; Guess et al., 2018).

First, although research finds evidence of a preference for likeminded information, studies also find that there is not a parallel bias against counter-attitudinal information (e.g., Garrett, 2009b, 2009a; Knobloch-Westerwick \& Meng, 2009; Kobayashi \& Ikeda, 2009). In other words, there is an asymmetry in selective exposure: the preference for attitude-consistent information is stronger than the desire of avoiding counter-attitudinal information (Garrett, 2009b). Hence, research reports considerable exposure to congenial information, but not to the exclusion of opposing information (Guess, 2018). Moreover, under certain conditions (e.g., when there is a need to defend the own views against others or to strive for accuracy), people will actively seek counter-attitudinal information and will find it useful to consume dissimilar or uncongenial media (Valentino, Hutchings, Banks, \& Davis, 2008).

Second, in the rich and complex digital media environment people have the possibility to use alternative cues to select information. Aside from brands, online media offer readers other shortcuts to information selection, such as the number of clicks on an article. People's choices and recommendations of news are also visible through social media platforms and may be used as cues for information selection (Messing \& Westwood, 2012). Several studies testify to the popularity of social recommendations as cues to information selection (Knobloch-Westerwick, Sharma, 
Hansen, \& Alter, 2005; Messing \& Westwood, 2012), which, in turn ,through "weak ties" may increase the probability of exposure to diverse information (Barberá, 2014).

Finally, the expectation that people will self-segregate ideologically is at odds with the observation that people tend to care little about politics (Converse, 1964). Politically low-motivated people will not be very likely to take costly action to select ideologically consistent information (Zaller, 1992). This should be especially true when the costs of avoiding heterogeneous information are high, as in a media environment characterized by weak boundaries (Brundidge, 2010).

Based on this discussion, we expect to find evidence of selective exposure at the individual level, but not at the expense of avoiding exposure to counter-attitudinal media. Hence, we do not expect partisan echo-chambers to prevail in online news consumption. This leads to the following two hypotheses:

(H1): Individuals spend more time in congenial media than in uncongenial media

(H2): Most people cross partisan lines in online news consumption

\section{Echo-chambers during major news events}

Under normal conditions we expect people to spend more time in congenial media but to engage as well in considerable cross-partisan media consumption. But what happens under exceptional conditions, for example, when major news events occur? With some exceptions (e.g. Guess, 2016), studies using digital trace data have not examined changes in regular news consumption behaviour, such as during major news events. Major news events are expected to increase news consumption and can consequently have an effect on partisan selective exposure. 
In particular, major news events might shape different active informationseeking strategies depending on the topic. The nature of topics may affect selective exposure differently (see e.g., Barbera et al. 2015) by activating different motivations (Kruglanski, 1999; Kunda, 1990; Guess, 2016). Political topics may be particularly likely to inspire selective exposure because they often trigger an affective response, which in turn propels partisans to engage in motivated reasoning (Stroud, 2008; Taber and Lodge, 2006). Motivated reasoning has been shown to have implications not only for information processing but also for information search. For example, Lodge, Taber, and Galonsky (1999) show a preference for congruent information during information search about political issues when subjects knew the likely valence of available information. In short, salient political news events such as an election would be expected to activate defence or partisan reinforcement motivations (Kruglanski, 1999, Guess, 2016) and thus to enhance partisan-media consumption.

In contrast, news events of a non-political character such as a plane crash might increase cross-cutting exposure by prompting a need for accuracy and factual information. Although accuracy-motivation effects on information search have been seen to be conditional on outcome performance and information utility (Valentino et al., 2009; Hart et al., 2009), some contend that it will lead to a more balanced information search (Jonas, Schulz-Hardt \& Frey, 2005; Chen, Lee \& Gil de Zúñiga, 2012). In contrast to searching for feelings of validation, a search for factual information "may necessitate casting a wide net, seeking out relatively objective sources, and avoiding partisan outlets" (Guess, 2016: 7). This leads to our third and fourth hypotheses:

(H3): Major news event of a highly salient political character will increase selective exposure. 
(H4): News events of a non-political character will increase cross-cutting media exposure

\section{Data and Methods}

Design. This study uses two interdependent datasets to investigate the prevalence of selective exposure in online news consumption in Spain. We use a twowave survey data to measure individuals' reported ideology and other political and demographic traits, and navigation data of a subset of these respondents $(\mathrm{N}=408)$ for a period extending from 26 January to 27 April 2015. During this period, several major news events occurred: a jihadist attack in Tunisia (19 March 2015), regional elections in Andalucia (22 March 2015) and the Germanwings plane crash on the French Pyrenees (24 March 2015). Our navigation dataset includes all sites visited from laptops throughout the study period, the date, the initiating and finishing time, and the total amount of time (in seconds) spent at each site. For our study, we targeted individuals that consumed a minimum of information online ${ }^{1}$ and who had their residence in all Spanish regions except for Catalonia ${ }^{2}$.

Panel. Respondents for our study were selected from an online survey pool that only includes users by invitation. A commercial provider recruits panellists-from which our sample was selected-through a multisource and closed process. This approach is aimed to guarantee the engagement of a broad range of socio-demographic profiles. To avoid duplications, users can only join the panel if they receive the personal invitation and after a double opt-in process ${ }^{3}$. Our sample targets a representation of the online population that consumes political information on the Internet and is based on those who initially opt to participate in the study rather than a probability sample. Hence, no estimates of sampling error can be calculated. 
Overall, our sample has characteristics that are different from the general population; yet, it matches the characteristics of the Spanish online population, which is the target population we aim to study. This population tends to be, on average, younger, more educated, more interested in politics, and more left-leaning than the general population (Robles, Molina, \& De Marco, 2012). Table A1 in the online Appendix compares the characteristics of our panel with the Spanish general population ${ }^{4}$.

Coding procedure. We used a dataset on navigation data on desktop devices for a period of 92 days, which contains a total of 1,024,026 URLs of visited sites corresponding to 79,071 domains for 408 unique users. These observed data for online navigation was filtered in order to identify news consumption for the top 42 most visited news outlets in Spain according to Alexa rankings ${ }^{5}$. Visits to outlets in our list of 42 totalled 40,683, which amounts to 3,97\% of total site visits during our period of study. The navigation data corresponding to the 42 news outlets was then collapsed to individual level data for the news outlets of interest, time spent in each outlet, total navigation time, and the number of page views.

Individuals who, during the tracking period, did not visit an outlet from our list of 42 were automatically excluded from the analysis. Out of 408 panellists, 388 visited at least one outlet on our list.

\section{Measurements}

Selective Exposure. Following other studies (e.g., An, Quercia, \& Crowcroft, 2013), we use an Index of Net Partisan Skew (NPS) to measure selective exposure. Our NPS Index results from subtracting the amount of time an individual is exposed to left leaning media from the amount of time he/she is exposed to right leaning media. Values that are close to 0 in this index indicate a balanced media diet, while positive values 
indicate a skew toward right leaning media, and negative values, a skew toward left leaning media. Table 1 reports the mean, standard deviation, minimum and maximum values of this and other key variables.

- Table 1 about here -

Given the large variance in this variable, we use the difference on the log scale as our main dependent variable:

$$
\mathrm{NPS}=\log (\mathrm{REi}+1)-\log (\mathrm{LEi}+1)
$$

Individuals' news exposure. To measure news exposure online, we relied on observable measures of exposure, and we used time of exposure to the 42 news outlets on our list. As an average people spent 221,53 seconds per day on our list of 42 news outlets, which represents $3.7 \%$ of the total time they spent navigating different sites online. However, as we can see in Table 1, there is a huge range of variation in time spent in news outlets among our panellists, running from 0 to 2,364 seconds per day.

Individuals' political leanings. To measure people's political leanings, we used ideological self-placement in a left-right scale from 0 (extreme left) to 10 (extreme right). As we can see in Table 1, the mean value of ideology in our sample is 4 with SD of 2.08, a value that is slightly to the left than the mean for the whole Spanish population ( $\mathrm{m}=4.4 ; \mathrm{SD}=2.1)$ (see Table $\mathrm{A} 1$ in the online Appendix)

Media slant. To measure ideological slant of the 42 news outlets considered in our study, we relied on reported media positions from the survey. Respondents provided the ideological position of each media outlet they usually visit (left, right, or neutral). We classified an outlet as partisan media if at least $50 \%$ of respondents perceive it as right- or left-leaning. Our classification is shown in the online Appendix (Table A2) ${ }^{6}$. In Table 1 we can see that almost half of the media included in our list of 42 are classified as partisan $(\mathrm{m}=0.51)$. More importantly, the list of partisan media includes the two 
major Spanish news outlets, El Mundo and El Pais, a result that matches previous classifications and research. This is consistent with the characteristics of the Spanish media system, which has been classified as polarized pluralism (Hallin \& Mancini, 2004), in contrast for example to liberal media systems such as the US, where, mainstream outlets and news portals tend to be neutral.

\section{Results}

Selective exposure (H1 and H2). Of all time devoted to online navigation people in our panel spent $3,7 \%$ of the time in news consumption ${ }^{7}$, and of all time devoted to news consumption they spent $60 \%$ of the time in partisan media. These figures testify to the marginal role of news consumption in people's online activities, which is consistent with the claim that people typically have a low interest in politics (Converse, 1964), and to the importance of partisan media in the Spanish Media System. Figure 1 shows the number of visits to neutral (Panel a) and partisan media (Panel b) by ideology weighted by the proportion of self-declared left and right wing people in the population. It shows that partisan media is more popular than neutral media for panellists from all ideological positions (even for those positioning themselves in the middle of the scale).

-- Figure 1 about here --

Hypothesis 1 expects to find differences in news consumption across ideologues given that left and right leaning individuals probably prefer media consistent with their political views. Panel c in Figure 1 plots visits to partisan media for all ideological positions (0-10), showing that partisans tend to visit more frequently media of their own political leanings. Panel d uses time spent in partisan media to plot NPS by ideology. Values around 0 indicate a balanced media diet (spending as much time in conservative 
as in liberal media), positive values denote a skew towards conservative media, and negative values imply a skew towards liberal media. As expected, for individuals located on the right (6-10) values are positive (except for those located at 9), even if not all values are expected to be different from 0 . Similarly, the observed means in the same Index for left-leaning individuals (0-4) are negative (except for individuals located at 4 on the scale), although again not all these values are expected to be different from 0 . This indicates that right leaning individuals spend more time in conservative media relative to their left-leaning counterparts, while self-declared leftists devote more time to liberal media relative to their conservative counterparts. However, there are differences across groups with regard to the extent of selective exposure: while mean values are clearly positive for right-wing individuals they are much closer to 0 for leftwing individuals. Moreover, most values for those situated in the left (0-4) are indistinguishable from 0 . This suggests that self-identified leftists are exposed to more cross-ideological media.

Despite these differences, we expect individuals to cross partisan lines in online news consumption (H2). To test $\mathrm{H} 2$ we first look at the distribution of our NPS Index. At one extreme, if people only consume likeminded media we should see a bimodal distribution with two peaks - one, for the left, centred on negative values, and the other, for the right, centred on positive values -- and no or little overlapping between the left and the right's distributions. At the other extreme, if media diets are balanced we should see a unimodal distribution centred on values around 0 and total or much overlapping between the left and right's distributions. Panel a in Figure 2 plots this index's distribution for the whole sample (line in black) and the two ideological groups (left=red line; right=blue line). 
It shows that the distribution for the whole sample is unimodal and centred on a value quite close to $0(\mathrm{~m}=0.20 ; \mathrm{SD}=1,27)$. Although the distributions for the left and the right are centred around negative and positive values, respectively, these values are also quite close to 0 (left $=-0.07, \mathrm{SD}=1.07$; right $=0.68, \mathrm{SD}=1.31)$, and there is considerable overlapping between these distributions. To test the robustness of these results we tested the normality of the three distributions using the Kolmogorov-Smirnov test; the results are included in Table A3 of the Online Appendix.

To get a better idea of the extent of cross-ideological exposure, in Panel $b$ we plot the time of exposure to right-wing media over time of exposure to left-wing media for all individuals in our sample visiting an outlet during the study period. This figure clearly shows that most people $(82,2 \%)$ spent time reading news from both sides of the political spectrum, and that individuals consuming news only from one side of the political spectrum are a minority $(14,1 \%)$. Moreover, a good number of people in the left (those lying above the diagonal line) spent more time on media from the other side than from their own. This stands in contrast to the behaviour of right-wing individuals, who typically spend more time on congenial media (most lie below the diagonal line).

Selective exposure under external shocks (H3 and H4). News supply determine the level of partisan news consumption from users. Major news events increase news consumption. Hypothesis 3 and 4 expect behaviour in news consumption to vary according to the nature of breaking news. We use exogeneous events that occurred during the fieldwork of the navigation study as a quasi experiment ${ }^{8}$ that allows to explore the behaviour of media consumption in exceptional situations such as those that happened on those days where three news events took place: Jihadist attack in Tunisia 19 March 2015; the electoral victory of the socialist Susana Diaz in Andalucian regional elections 22 March 2015; and Germanwings 24 March 2015. The first two 
events are of an overtly political nature (and the second of an overtly partisan nature), while the last one is not ${ }^{9}$.

To try to verify these hypotheses, for each day of the study, we calculate the average value of navigation time in liberal and conservative media (i.e., the NPS value) for users self-defined as right and left leaners ${ }^{10}$. Panel a in Figure 3 shows the evolution of selective exposure for the two groups of users.

-- Figure 3 about here --

We define $\mathrm{D}$ as the difference between the mean values, on a logarithmic scale, of the values of selective exposure for the two groups of users, $n$ being the number of consumed media by users self-defined as right leaning, and $\mathrm{m}$, the number of consumed media by users self-defined as left leaning.

$$
\mathrm{D}=\left(\frac{\sum_{\mathrm{i}=1}^{\mathrm{n}} \log \left(\mathrm{t}_{\mathrm{MR}}^{\mathrm{i}}+1\right) \cdot \log \left(\mathrm{t}_{\mathrm{ML}}^{\mathrm{i}}+1\right)}{\mathrm{n}}\right)-\left(\frac{\sum_{\mathrm{j}=1}^{\mathrm{m}} \log \left(\mathrm{t}_{\mathrm{MR}}^{\mathrm{j}}+1\right)-\log \left(\mathrm{t}_{\mathrm{ML}}^{\mathrm{j}}+1\right)}{\mathrm{m}}\right)
$$

In order to assess the extent to which the daily behaviour moves away from the values that would be expected in terms of statistical fluctuation we calculate the difference between the daily navigation values and the mean value of the time series $\left(D_{M}=0.79, \sigma_{D}=0.19\right)$, and express the difference in terms of standard deviations over the difference to the average value.

$$
D_{\sigma}=\frac{D-D_{M}}{\sigma_{D}}
$$

The graphic representation (Panel b in Figure 3) allows to observe, in general terms, a parallel evolution of the level of selective exposure of the two groups of users. However, the representation shows periods with abnormal behaviour in terms of statistical fluctuations in overall behaviour.

The positive values of $D_{\sigma}$ correspond to days where the selective exposure levels of the two groups are more differentiated (more partisan), while negative values indicate 
more similar consumption patterns (less partisan). The most significant pattern in terms of anomalous behaviour is comprised around the period between 17 March and 3 April.

The days after the attack in Tunisia, and the electoral victory of Susana Diaz in the Andalusian elections intensifies the differentiated behaviour of the two groups of users, reaching a significant difference of more than 2 sigma. The selective exposure of each group is accentuated and has its maximum the day of the Germanwings plane accident (24 March). From that moment on, a significant change of trend is observed, during which the difference between the values of selective exposure is gradually reduced, indicating more equitable behaviours between the two groups of users.

The results obtained thus seem to lead us to the conclusion that a spectacular news event, without a marked political character, such as that of the Germanwings aircraft, increases the difference between the selective exposure values of the two groups of users the day of the event as can be seen in the value of $D_{\sigma}$. After that a significant reduction in selective exposure appears as we can see in $D_{\sigma}$ values between 25 March and 3 April 2015.

The cause of these variations in selective exposure levels cannot be attributed to a change in user behaviour caused by the nature of news, but rather to an accentuation of the consumption trends of each group. To verify this we use the total navigation time in media of the set of users. The total navigation time of users has been estimated in a window of 5 days and the difference in sigma with respect to the average navigation time has been estimated in a procedure analogous to the previous one.

Coinciding with the period in which the quasi experiment takes place we observe that media consumption, is effectively much higher than the average consumption. Comparing the evolution of the time spent in media consumption and the evolution of the D value (both in absolute terms and in terms of standard deviations from the mean value) 
a very strong correlation is obtained between time in media consumption and the values of selective exposure for users in the two sample groups $(\rho=0.682 ; p<0,001)$. This correlation increases in the time lapse of the natural experiment between 19 March and 4 April $(\rho=0.939 ; p<0,001)$.

In conclusion, the accentuation and reduction in the global values of selective exposure is a consequence not of a change in the pattern of consumption of users, but of the fact that greater media consumption in both groups is associated with more skewed partisan behaviour in the consumption of media.

\section{Discussion and conclusions}

The increasing number of news outlets reflecting a wide range of ideological views and the possibilities to personalize and filter content made available by the web have led many to expect that people would inhabit partisan echo-chambers when consuming news online. Although this hypothesis has received considerable attention from scholars, especially in the US, we have submitted it to a tougher test by studying the Spanish case, a country known by its high political parallelism. In line with other studies using digital trace data, this study finds no evidence of partisan echo-chambers in online news consumption. However, our results are driven by different patterns in media consumption.

Most analyses focusing in the US show that low levels of selective exposure are driven by most consumption being concentrated on a handful of mainstream news portals, which tend to be centrist (Gentzkow \& Shapiro, 2011; Guess, 2016; Flaxman et. al, 2016; Nelson \& Webster, 2017; Weeks et al., 2016). In contrast, given the partisan nature of the Spanish media system, we find that much of the exposure to counter attitudinal information results from crossing partisan lines in media consumption, 
especially from those in the left. Our results suggest that people take advantage of the diversity of views online rather than avoiding different opinions even in polarized media systems. They confirm both motivational explanations on the need to seek counter-attitudinal information and will find it useful to consume dissimilar or uncongenial media (Valentino, Hutchings, Banks, \& Davis, 2008) and arguments based on the structure of media environments where avoiding heterogeneous information is costly (Brundidge, 2010). Furthermore, because here we only consider consumption of partisan media and not of neutral media, which is also quite extensive (36\% of visits are to neutral media), our study probably underestimates the amount of cross-cutting exposure that exists in a partisan media system such as the Spanish one. Our results thus challenge the partisan echo-chambers hypothesis and testify further to its myth (Nelson \& Webster, 2017)

Our study however suggests that selective exposure intensifies as news consumption increases. We find that the values of overall news consumption change with the nature of major news events, although this has less to do with the type of event than with the amount of interest it arises. Overall, our study uncovers that users polarize their behaviour along party lines as the level of news consumption increases, a result that is consistent with findings showing at the individual level that political interest and news consumption increases partisan behaviour (An et al., 2013; Prior, 2008, 2013; Stroud, 2011, 2008) thus increasing the need for validation and activating defence or partisan reinforcement motivations (Kruglanski, 1999, Guess, 2016). These findings suggest that it is vital that future studies consider both the nature of the media system and the type of news when studying partisan selective exposure.

This study is not exempt from several limitations. The first limitation is that navigation data excludes mobile devices and thus limits the scope of our results because 
studies show that people increasingly use mobile devices for online news consumption. The second limitation has to do with the characteristics of our panel. Some contend that people accepting to be tracked are less concerned about privacy (Callegaro et al., 2014). In turn, this might correlate with other attitudes, such as curiosity and openness, biasing our findings. Differences in these particular attitudes may be a serious concern, with no possible way around this problem because tracking people's navigation behaviour requires consent. Finally, a third important limitation of this study is that exposure is measured using the choice of media outlets, not content. This may also be a problem for our results because some of the diversity we find might be driven not by consumption of news but of entertainment, although we minimize this problem by focusing on partisan media. 


\section{Footnotes}

${ }^{1}$ We used four filter questions-from less to more restrictive. The less restrictive question asked people if they followed current events or read news online. A positive answer in any of the four questions was enough to include the individual in our panel. Only four people were filtered out, which proves the inclusiveness of our questions and rules out potential problems of selection bias. The questionnaire can be accessed here: https://www.dropbox.com/s/j4j4wyog32wujxs/20160113 Questionnaire Selective\%20 Exposure $\% 20$ and $\% 200$ nline $\% 20$ Media.pdf?dl $=0$.

${ }^{2}$ We excluded Catalonia from our study because, in contrast to Spanish politics, conflict is multidimensional. Adding dimensions would have unnecessarily complicated a study about segregation. In addition, during the period of this study, the second dimension was especially salient in Catalonia, as elucidated by the rise of the proindependence movement.

${ }^{3}$ More information about the recruitment process and the sample sources can be found in the online Appendix that can be accessed here: https://www.dropbox.com/s/0t12ssb0c7tc406/Online_Appendix.docx?dl=0

${ }^{4}$ Table A1 is included in the online Appendix

${ }^{5}$ We selected the top 42 most visited news outlets reported by Alexa because they represent an important percentage of the total audience for news according to both Alexa and ComScore. The strong correlation (0.906) between media's position in both these lists-Alexa and ComScore-proves the accuracy of our media sample. Our list of 42 media includes $99,85 \%$ of all reported visits to online news outlets in our sample.

${ }^{6}$ The online Appendix that can be accessed here: https://www.dropbox.com/s/0568qr11gtsu17h/20181228 Online_Appendix.docx?dl=0 
${ }^{7}$ Most navigation time corresponds to the big hubs such as commercial sites, entertainment, sport and yellow press and magazines.

${ }^{8} \mathrm{We}$ use a simple interrupted time series design by considering three major news events. This is a quasi-experimental design as we do not control over exposure to the news stories on these events i.e, we do not expect exposure to be random (Campbell \& Stanley, 1963). However, this approach describes intra-individual differences between pre and post-event behaviour, and increases the possibility to rule out that individuals change their media consumption behaviour for some other reason than the emergence of the major news events.

${ }^{9}$ There are other differences between these news events. For example, two of these events are sudden major news events, while regional elections in Andalucia are not. Table A4 in the online appendix provides information of the coverage (in number of news appeared in all Spanish media) of these news events.

${ }^{10}$ Given the volatility of the measures obtained for this value (due in part to having an $\mathrm{N}$ limited to 408 individuals) we chose to study the trend in users' media consumption from a sliding window of the temporal sequence (Gaber, Zaslavsky, \& Krishnaswamy, 2005; Ippel, Kaptein, \& Vermunt, 2017). The selection of the value of the amplitude of the window is estimated in 5 days $(-2$ and +2$)$. 


\section{References}

Allcott, H., \& Gentzkow, M. (2017). Social media and fake news in the 2016 election. Journal of Economic Perspectives, 31(2), 211-236.

An, J., Quercia, D., \& Crowcroft, J. (2013). Fragmented social media: a look into selective exposure to political news. En Proceedings of the 22nd International Conference on World Wide Web (pp. 51-52).

Bakshy, E., Messing, S., \& Adamic, L. A. (2015). Exposure to ideologically diverse news and opinion on Facebook. Science, 348(6239), 1130-1132.

Barberá, P. (2014). How social media reduces mass political polarization. Evidence from Germany, Spain, and the US. Job Market Paper, New York University, 46.

Barberá, P., Jost, J. T., Nagler, J., Tucker, J. A., \& Bonneau, R. (2015). Tweeting from left to right: Is online political communication more than an echo chamber?. Psychological science, 26(10), 1531-1542.

Bennett, W. L., \& Iyengar, S. (2008). A new era of minimal effects? The changing foundations of political communication. Journal of Communication, 58(4), $707-731$.

Brundidge, J. (2010). Encountering "difference" in the cntemporary public sphere: The contribution of the Internet to the heterogeneity of political discussion networks. Journal of Communication, 60(4), 680-700.

Callegaro, M., Baker, R. P., Bethlehem, J., Göritz, A. S., Krosnick, J. A., \& Lavrakas, P. J. (2014). Online panel research: a data quality perspective. John Wiley \& Sons.

Campbell, D. T., \& Stanley, J. C. (1963). Experimental and quasi-experimental designs for research on teaching. American Educational Research Association.

Chaffee, S. H., \& Metzger, M. J. (2001). The end of mass communication? Mass 
communication \& society, 4(4), 365-379.

Converse, P. E. (1964). The Nature of Belief Systems in Mass Publics. In Ideology and Discontent, ed. David Apter. New York: Free Press.

Del Vicario, M., Bessi, A., Zollo, F., Petroni, F., Scala, A., Caldarelli, G., ... Quattrociocchi, W. (2016). The spreading of misinformation online. Proceedings of the National Academy of Sciences, 113(3), 554-559.

Dubois, E., \& Blank, G. (2018). The echo chamber is overstated: the moderating effect of political interest and diverse media. Information, Communication \& Society, 21(5), 729-745.

Dvir-Gvirsman, S., Tsfati, Y., \& Menchen-Trevino, E. (2016). The extent and nature of ideological selective exposure online: Combining survey responses with actual web log data from the 2013 Israeli Elections. New Media \& Society, 18(5), 857-877.

Feldman, L., Stroud, N. J., Bimber, B., \& Wojcieszak, M. (2013). Assessing selective exposure in experiments: The implications of different methodological choices. Communication Methods and Measures, 7(3-4), 172-194.

Festinger, L. (1962). A theory of cognitive dissonance (Vol. 2). Stanford University Press.

Flaxman, S., Goel, S., \& Rao, J. (2016). Filter bubbles, echo chambers, and online news consumption. Public Opinion Quarterly, 80(S1), 298-320.

Gaber, M. M., Zaslavsky, A., \& Krishnaswamy, S. (2005). Mining data streams: a review. ACM Sigmod Record, 34(2), 18-26.

Garrett, R. K. (2009a). Echo chambers online?: Politically motivated selective exposure among Internet news users1. Journal of Computer-Mediated Communication, $14(2), 265-285$.

Garrett, R. K. (2009b). Politically motivated reinforcement seeking: Reframing 
the selective exposure debate. Journal of Communication, 59(4), 676-699.

Gentzkow, M., \& Shapiro, J. M. (2011). Ideological Segregation Online and Offline. The Quarterly Journal of Economics, 126(4), 1799-1839.

Guess, A. (2016). Media Choice and Moderation: Evidence from Online Tracking Data .

Guess, A., Nyhan, B., Lyons, B., Reifler, J. (2018). Avoiding echo-chambers about echo-chambers: Why selective exposure to political news is less prevalent than you think. Knight Foundation.

Hallin, D. C., \& Mancini, P. (2004). Comparing media systems: Three models of media and politics. Cambridge: Cambridge University Press.

Hannak, A., Sapiezynski, P., Molavi Kakhki, A., Krishnamurthy, B., Lazer, D., Mislove, A., \& Wilson, C. (2013). Measuring personalization of web search. Proceedings of the 22nd international conference on World Wide Web (pp. 527-538). ACM.

Hart, W., Albarracín, D., Eagly, A. H., Brechan, I., Lindberg, M. J., \& Merrill, L. (2009). Feeling validated versus being correct: a meta-analysis of selective exposure to information. Psychological bulletin, 135(4), 555.

Holbert, R. L., Hmielowski, J. D., \& Weeks, B. E. (2012). Clarifying relationships between ideology and ideologically oriented cable TV news use: A case of suppression. Communication Research, 39(2), 194-216.

Hollander, B. A. (2008). Tuning out or tuning elsewhere? Partisanship, polarization, and media migration from 1998 to 2006. Journalism \& Mass Communication Quarterly, 85(1), 23-40.

Ippel, L., Kaptein, M., \& Vermunt, J. (2017). Dealing with Data Streams: an Online, Row-by-Row, Estimation Tutorial. Methodology: European Journal of 
Research Methods for the Behavioral and Social Sciences.

Jonas, E., Schulz-Hardt, S., \& Frey, D. (2005). Giving advice or making decisions in someone else's place: The influence of impression, defense, and accuracy motivation on the search for new information. Personality and Social Psychology Bulletin, 31(7), 977-990.

Iyengar, S., \& Hahn, K. S. (2009). Red media, blue media: Evidence of ideological selectivity in media use. Journal of Communication, 59(1), 19-39.

Knobloch-Westerwick, S., \& Meng, J. (2009). Looking the other way: Selective exposure to attitude-consistent and counterattitudinal political information. Communication Research, 36(3), 426-448.

Knobloch-Westerwick, S., Sharma, N., Hansen, D. L., \& Alter, S. (2005). Impact of popularity indications on readers' selective exposure to online news. Journal of broadcasting \& electronic media, 49(3), 296-313.

Kobayashi, T., \& Ikeda, K. (2009). Selective exposure in political web browsing: Empirical verification of 'cyber-balkanization' in Japan and the USA. Information, Communication \& Society, 12(6), 929-953.

Kruglanski, A. W. (1999). Motivation, cognition, and reality: Three memos for the next generation of research. Psychological Inquiry, 10(1), 54-58.

Kunda, Z. (1990). The case for motivated reasoning. Psychological bulletin, $108(3), 480$.

Lodge, M., Taber, C., \& Galonsky, A. C. (1999, September). The political consequences of motivated reasoning: Partisan bias in information processing. In Annual Meeting of the American Political Science Association. Atlanta.

Messing, S., \& Westwood, S. J. (2012). Selective exposure in the age of social media: Endorsements trump partisan source affiliation when selecting news online. 
Communication Research, 41(8), 1042-1063.

Metzger, M. J., Hartsell, E. H., \& Flanagin, A. J. (2015). Cognitive dissonance or credibility? A comparison of two theoretical explanations for selective exposure to partisan news. Communication Research, 0093650215613136.

Nelson, J. L., \& Webster, J. G. (2017). The Myth of Partisan Selective Exposure: A Portrait of the Online Political News Audience. Social Media+ Society, 3(3), 2056305117729314.

Pariser, E. (2011). The filter bubble: What the Internet is hiding from you. Penguin UK.

Prior, M. (2008). Are hyperlinks 'weak ties'? En J. Turow \& L. Tsui (Eds.), The hyperlinked society: questioning connections in the Digital Age (pp. 227-249).

University of Michigan Press Ann Arbor, MI.

Prior, M. (2009). The Immensely Inflated News Audience: Assessing Bias in Self-Reported News Exposure. Public Opinion Quarterly, 73(1), 130-143.

Prior, M. (2013). Media and Political Polarization. Annual Review of Political Science, 16(101-127), 101-127.

Robles, J. M., Molina, Ó., \& De Marco, S. (2012). Participación política digital y brecha digital política en España. Un estudio de las desigualdades digitales. Arbor, 188(756), 795-810.

Stroud, Natalie J. (2011). Niche news: The politics of news choice. Oxford: Oxford University Press.

Stroud, Natalie J. (2008). Media use and political predispositions: Revisiting the concept of selective exposure. Political Behavior, 30(3), 341-366.

Sunstein, C. R. (2009). Republic. com 2.0. Princeton University Press.

Taber, C. S., \& Lodge, M. (2006). Motivated skepticism in the evaluation of 
political beliefs. American Journal of Political Science, 50(3), 755-769.

Thorson, K., \& Wells, C. (2015). Curated flows: A framework for mapping media exposure in the digital age. Communication Theory, 26(3), 309-328.

Thurman, N., \& Schifferes, S. (2012). The future of personalization at news websites: lessons from a longitudinal study. Journalism Studies, 13(5-6), 775-790.

Valentino, N. A., Hutchings, V. L., Banks, A. J., \& Davis, A. K. (2008). Is a worried citizen a good citizen? Emotions, political information seeking, and learning via the internet. Political Psychology, 29(2), 247-273.

Valentino, N. A., Banks, A. J., Hutchings, V. L., \& Davis, A. K. (2009).

Selective exposure in the Internet age: The interaction between anxiety and information utility. Political Psychology, 30(4), 591-613.

Valeriani, A., \& Vaccari, C. (2015). Accidental exposure to politics on social media as online participation equalizer in Germany, Italy, and the United Kingdom. New Media \& Society, 18(9), 1857-1874.

Weeks, B. E., Ksiazek, T. B., \& Holbert, R. L. (2016). Partisan enclaves or shared media experiences? A network approach to understanding citizens' political news environments. Journal of Broadcasting \& Electronic Media, 60(2), 248-268.

Wojcieszak, M., Bimber, B., Feldman, L., \& Stroud, N. J. (2016). Partisan News and Political Participation: Exploring Mediated Relationships. Political Communication, 33(2), 241-260.

Zaller, J. (1992). The nature and origins of mass opinion. Cambridge: Cambridge University Press.

Zuiderveen Borgesius, F., Trilling, D., Moeller, J., Bodó, B., de Vreese, C. H., \& Helberger, N. (2016). Should we worry about filter bubbles? 